\title{
Validation of an Ergonomic Method of Withdrawing 99mTc-Radiopharmaceuticals
}

\author{
Sandy Blondeel-Gomes ${ }^{1}$, Solène Marie ${ }^{1}$, Sabrina Loyeau ${ }^{2}$, Julien Fouque ${ }^{1}$, François Lokiec ${ }^{1}$, and Olivier Madar ${ }^{1}$ \\ ${ }^{1}$ Department of Radio-Pharmacology, Institut Curie Hospital, Paris, France; and ${ }^{2}$ Operational Hygiene Team, Institut Curie Hospital, \\ Paris, France
}

The main objective of the present work was to ensure the quality of radiopharmaceutical syringes withdrawn with a system consisting of a spinal needle and an obturator IN-Stopper. Methods: Visual examinations and physicochemical tests were performed at baseline and $4 \mathrm{~h}$ for ${ }^{99 \mathrm{mT}} \mathrm{Tc}$-albumin nanocolloid and at baseline and $7 \mathrm{~h}$ for ${ }^{99 \mathrm{mT}} \mathrm{Tc}$-eluate, ${ }^{99 \mathrm{mT}} \mathrm{Tc}$-hydroxymethylene diphosphonate, and ${ }^{99 m}$ Tc-human serum albumin. Microbiologic validation was performed according to the European pharmacopoeia. Fingertip radiation exposure was evaluated to confirm the safety of the system. Results: The results showed stable visual and physicochemical properties. The integrity of the connector was not affected after 30 punctures (no cores). No microbiologic contamination was found on tested syringes. Concerning radiation safety, no overexposure was reported with the system. Conclusion: The system could be used 30 times. The stability of syringes withdrawing radiopharmaceuticals with this method is guaranteed up to $4 \mathrm{~h}$ for ${ }^{99 \mathrm{mT}} \mathrm{Tc}$-albumin nanocolloid and $7 \mathrm{~h}$ for ${ }^{99 \mathrm{~m} T c} \mathrm{~T}$-eluate, 99mTc-hydroxymethylene diphosphonate, and 99mTc-human serum albumin.

Key Words: quality assurance; ${ }^{99 \mathrm{mT}} \mathrm{T}$; radiopharmaceuticals; radiopharmacy

J Nucl Med Technol 2018; 46:49-52

DOI: $10.2967 /$ jnmt.117.199133

$\mathbf{T}$ o reduce personal exposure to $\gamma$-radiation, ${ }^{99 \mathrm{~m}} \mathrm{Tc}$ radiopharmaceutical vials are placed in tungsten shields with lead glass. Doses are usually prepared by reversal of the vial in order to withdraw the prescribed dose in a syringe. Shielded vials weigh between $900 \mathrm{~g}$ and $2 \mathrm{~kg}$. Syringes are also manipulated with a shield device whose weight is almost $200 \mathrm{~g}$. Because of the heaviness of the material, repetition of these actions induces serious physical demands on the staff.

In our department, a method avoiding reversal has been validated for ${ }^{18} \mathrm{~F}$-radiopharmaceutical dispensing. It consists of introducing a spinal needle connected to a membrane obturator IN-Stopper (B. Braun) (1) into the vial. The

\footnotetext{
Received Jul. 11, 2017; revision accepted Oct. 5, 2017.

For correspondence or reprints contact: Sandy Blondeel-Gomes, Department of Radio-Pharmacology, Institut Curie, Hospital, 26 rue d'Ulm, 75005 Paris, France.

E-mail: sandy.blondeel@curie.fr

Published online Nov. 10, 2017.

COPYRIGHT (c) 2018 by the Society of Nuclear Medicine and Molecular Imaging.
}

length of the needle allows for withdrawal of the totality of the liquid contained in the vial without reversal. The doses are dispensed by inserting the assembled needle and syringe into the obturator.

The use of the spinal needle and IN-Stopper would improve ergonomics, but a validation must be performed to guarantee the quality of the syringes drawn. Several interactions between medical device and radiopharmaceutical have been amply described (2), and process validation is necessary (3).

According to the manufacturer's recommendations, the obturator in the stopper can be punctured only 10 times by a 0.6-mm-diameter needle, which corresponds to our practice for ${ }^{18} \mathrm{~F}$-radiopharmaceuticals. ${ }^{99 \mathrm{~m}} \mathrm{Tc}$-radiopharmaceutical preparations are multidose vials, and in our department, 10 doses could be filled from one vial. The recommended number of uses of the connector would be exceeded because of readjustment of the dose needed to obtain the prescribed dose. Thus, the obturator integrity is not guaranteed, and a microbiologic risk exists. To ensure radiation safety, an evaluation is also necessary before routine use by staff.

The use of this method to withdraw ${ }^{99 m}$ Tc-radiopharmaceutical preparations (up to now used for ${ }^{18} \mathrm{~F}$-radiopharmaceuticals) requires a visual examination, a physicochemical and microbiologic validation, and a radiation safety evaluation.

\section{MATERIALS AND METHODS}

Tests were performed in a class A shielded hood (Tema Sinergie).

To limit the risk that rubber cores would form, a Quincke needle (Vygon), 18-gauge and $90 \mathrm{~mm}$ in length, was put into the vial's cap using the stylet. The stylet was removed and an obturator top, INStopper, was connected to the spinal needle. The system is represented in Figure 1. The dose was withdrawn into a syringe (Plastipak; BD Medical) connected to a needle, Microlance 3 (Becton Dickinson), $25 \mathrm{~mm}$ in length and 23-gauge, by piercing the rubber part of the connector. Shielded vials (Medisystem) weigh $915 \mathrm{~g}$ and shielded syringes (Medisystem) $172 \mathrm{~g}$.

\section{Visual Examination and Physicochemical Controls}

The system was tested with the following solutions: ${ }^{99 m} \mathrm{Tc}-$ pertechnetate eluate, ${ }^{99 \mathrm{~m} T c-h y d r o x y m e t h y l e n e ~ d i p h o s p h o n a t e ~}$ (99mTc-HMDP) (Osteocis; IBA Molecular), ${ }^{99 m} \mathrm{Tc}-$ albumin nanocolloid (Nanocoll; GE Healthcare), and ${ }^{99 m}$ Tc-human serum albumin ( $\left.{ }^{99 \mathrm{~m}} \mathrm{Tc}-\mathrm{HSA}\right)$ (Vasculocis; IBA Molecular). 


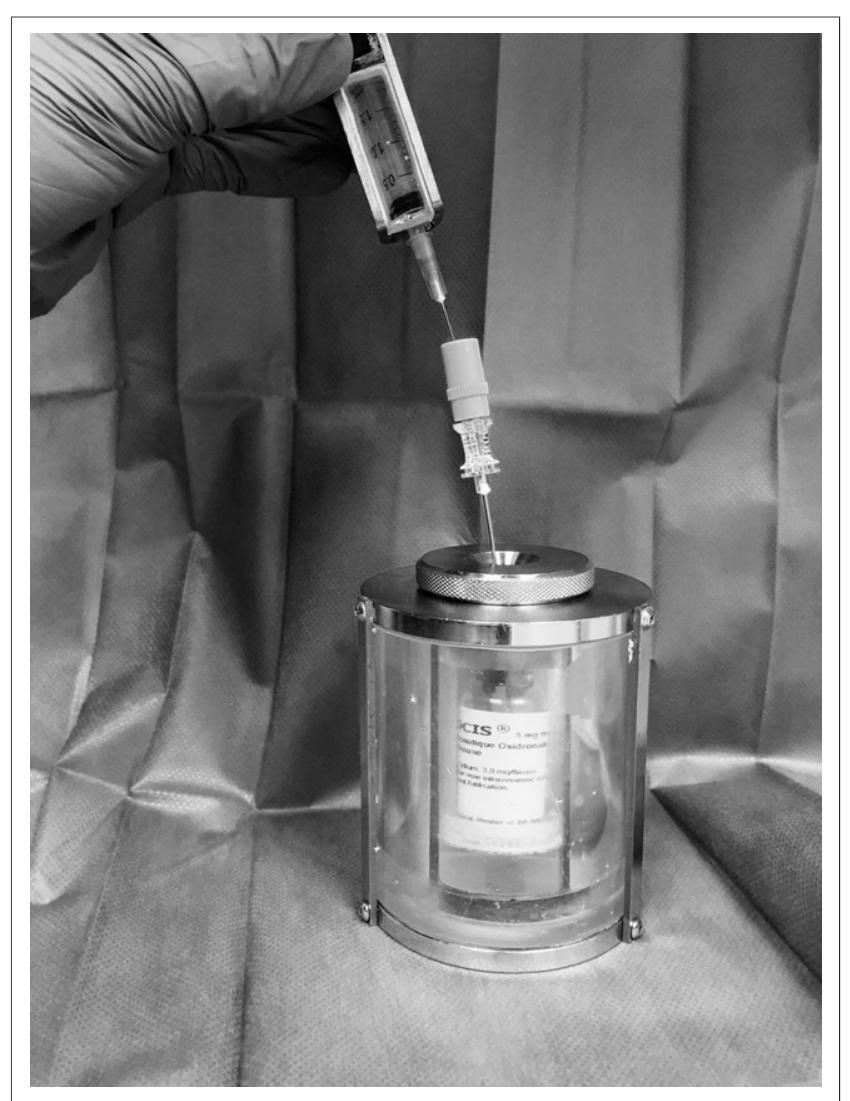

FIGURE 1. Photo of withdrawal system: spinal needle assembled to membrane obturator IN-Stopper.

${ }^{99 \mathrm{~m}} \mathrm{Tc}$-sodium pertechnetate was eluted from a Drytec ${ }^{99} \mathrm{Mo} /{ }^{99 \mathrm{~m}} \mathrm{Tc}$ generator (GE Healthcare). The ${ }^{99 \mathrm{~m}} \mathrm{Tc}$-radiopharmaceuticals were prepared following the manufacturer's instructions. The studied system was introduced into vials before the content for first quality control test was withdrawn (baseline). The stability study was performed for $7 \mathrm{~h}$ for ${ }^{99 \mathrm{~m} T c-e l u a t e}(n=5),{ }^{99 \mathrm{mTc}-H M D P}(n=5)$, and ${ }^{99 \mathrm{~m}} \mathrm{Tc}-\mathrm{HSA}(n=5)$ and for $4 \mathrm{~h}$ for ${ }^{99 \mathrm{~m}} \mathrm{Tc}$-albumin nanocolloid $(n=$ 5 ). Luer slip connector syringes with a capacity of $1 \mathrm{~mL}$ (Plastipak) were used for sampling. Vials were stored at room temperature throughout the study. Organoleptic specifications detailed in the product-characteristic summary were checked by a visual examination. $\mathrm{pH}$ was determined by $\mathrm{pH}$ indicator strips (MColorpHast; Merck Millipore).

Radiochemical purity was tested by instant thin-layer chromatography. For ${ }^{99 \mathrm{~m}}$ Tc-pertechnetate eluate, ${ }^{99 \mathrm{~m}} \mathrm{Tc}-\mathrm{HSA}$ (4), and ${ }^{99 \mathrm{~m} T c-a l b u m i n}$ nanocolloid (5), the method required Whatman 31ET strips (Clerad; Biodex) and acetone as solvent (GPR Rectapur; VWR). Concerning ${ }^{99 \mathrm{~m}} \mathrm{Tc}-\mathrm{HMDP}$, the radiochemical purity was determined by a 2 -strip procedure (5): instant thin-layer chromatography-silica gel strips and distilled water as solvent were used to determine the percentage of reduced or hydrolyzed ${ }^{99 \mathrm{~m}} \mathrm{Tc}$, and Whatman $31 \mathrm{ET}$ strip and acetone as solvent were used to evaluate the percentage of free pertechnetate.

After migration of the solvent, paper strips were scanned with a sodium iodine crystal detector on a MiniGITA Star (Elysia Raytest). Chromatograms were recorded and analyzed with GINAStar thin-layer chromatography (version 5.8).

\section{Visual Examination of IN-Stopper Connector}

The IN-Stopper membrane was examined for integrity and for the absence of small cores or plugs.

\section{Sterility Test}

The sterility tests were performed on 3 different days by 3 different operators. The rubber cap of the growth medium vial was cleansed with a chlorhexidine-sterilized pad before being punctured by the system. Each day, 10 syringes per operator (total, 30) were prepared with the spinal needle and IN-Stopper up to $7 \mathrm{~h}$ after the introduction of the system into the growth medium vial. Syringes were withdrawn at regular time intervals. Each 2.5-mL Luer slip connector syringe was prepared following the same protocol to simulate volume readjustment: IN-Stopper connector cleaned with chlorhexidine-sterilized pad; $1.5 \mathrm{~mL}$ of broth withdrawn into syringe; needle removed and then inserted; $1 \mathrm{~mL}$ withdrawn into growth medium vial; needle removed and then inserted; $0.5 \mathrm{~mL}$ of broth withdrawn into syringe (final volume, $1 \mathrm{~mL}$ ); needle disassembled and then assembled with 0.22-nm Perifix filter (B. Braun); hood air withdrawn; and filter disassembled and then assembling with a cap (Vygon).

The growth medium vials were stored at room temperature during the study.

Experimental conditions were validated by a positive control and a negative control. The positive control syringes were inoculated with Staphylococcus aureus and Aspergillus brasiliensis growth. Sterile water was used for the negative control. A positive transport sample and a negative transport sample were also prepared in syringes to validate transport conditions.

Each day, syringes were collected by Bioclin Laboratory. Microbiologic cultures were performed by Bioclin Laboratory according to the European Pharmacopoeia, 8th edition (6). Samples (spinal needle and IN-Stopper tests, positive control, negative control, positive transport sample, negative transport sample) were divided into 2 equal parts and directly inoculated onto 2 different media. Fluid thioglycolate medium and soybean casein digest broth were incubated at $30^{\circ} \mathrm{C}-35^{\circ} \mathrm{C}$ and at $20^{\circ} \mathrm{C}-25^{\circ} \mathrm{C}$, respectively, for $14 \mathrm{~d}$, with the exception of the positive control, which was incubated for $5 \mathrm{~d}$.

\section{Fingertip Radiation Exposure and Contamination}

A fingertip radiation dosimeter, Nuclear Educational Dosimeter (Unfors Instruments), was attached to the distal medial aspect of the index finger and covered with a pair of latex examination gloves. Tests were performed on the right index finger and then on the left index finger of each operator.

Dosimetry was measured and compared for 3 operators for both procedures: a routine process involving disinfection of the vial cap followed by reversal withdrawal of one syringe, and a spinal needle/obturator process consisting of insertion of the system into

TABLE 1

Activity and Volumes of Preparations

\begin{tabular}{ccc}
\hline Agent & Activity $(\mathrm{MBq})$ & \multicolumn{1}{c}{ Volume $(\mathrm{mL})$} \\
\hline 99mTc-HMDP & $10,930(10,220-11,600)$ & $5.0(5.0-5.0)$ \\
99mTc-HSA & $2,068(1,995-2,376)$ & $3.52(3.3-3.7)$ \\
99mTc-albumin & $1,634(1,368-1,814)$ & $4.78(4.8-5.0)$ \\
nanocolloid & & \\
\hline
\end{tabular}

Data are mean followed by range in parentheses. 
TABLE 2

Physicochemical Results for Controls

\begin{tabular}{|c|c|c|c|c|c|c|}
\hline \multirow[b]{2}{*}{ Agent } & \multicolumn{3}{|c|}{$\mathrm{pH}$} & \multicolumn{3}{|c|}{ Radiochemical purity (\%) } \\
\hline & Conformity & Baseline & 4 or $7 h^{*}$ & Conformity & Baseline & 4 or $7 h^{*}$ \\
\hline $\begin{array}{l}99 \mathrm{~m} \text { Tc-pertechnetate eluate } \\
\quad(n=5)\end{array}$ & $4-8$ & $5.84(5.8-6.0)$ & $5.80(5.8-5.8)$ & $>95 \%$ & 99.63 (99.37-99.99) & 99.56 (99.34-99.99) \\
\hline 99mTc-HMDP $(n=5)$ & $5-7$ & $5.36(5.0-5.5)$ & $5.36(5.0-5.5)$ & $>95 \%$ & 99.63 (99.34-99.79) & 99.05 (98.42-99.57) \\
\hline 99mTc-HSA $(n=5)$ & $2-6.5$ & $5.4(5.0-6.0)$ & $5.4(5.0-6.0)$ & $>95 \%$ & 99.70 (99.05-99.99) & 99.51 (99.13-99.76) \\
\hline $\begin{array}{l}99 \mathrm{~m} \text { Tc-albumin nanocolloid } \\
(n=5)\end{array}$ & $4-7$ & $5.0(5.0-5.0)$ & $5.0(5.0-5.0)$ & $>95 \%$ & 99.55 (99.31-99.99) & 99.67 (99.10-99.99) \\
\hline
\end{tabular}

${ }^{*} 7 \mathrm{~h}$ for ${ }^{99 m}$ Tc-pertechnetate eluate, ${ }^{99 m}$ Tc-HMDP, and ${ }^{99 m} \mathrm{Tc}-\mathrm{HSA}$, and $4 \mathrm{~h}$ for ${ }^{99 m}$ Tc-albumin nanocolloid.

Data are mean with or without range in parentheses.

the vial followed by disinfection of the obturator and withdrawal of one syringe. The vials used for the tests were filled with ${ }^{99 \mathrm{~m}} \mathrm{Tc}-$ pertechnetate eluate. Vials were measured using a dose calibrator, CRC 25R (Capintec), to calculate the received dose per megabecquerel for each hand.

Spillage and radioactive contamination were also studied. A contamination detector, LB 124 (Berthold), was used to measure radioactivity on shielded hood, gloves, vials, and syringe shields. Areas that registered more than twice the previously determined background level were considered contaminated.

\section{RESULTS}

\section{Characteristics of Tested Preparation}

The recommendations of the product-characteristic summary were followed for all preparations. Table 1 summarizes all preparation characteristics (activity and volume).

\section{Visual Examination and Physicochemical Controls}

The eluates and the preparation remained limpid and free from rubber coring. The physicochemical results are specified in Table 2. The results met the specifications of the productcharacteristic summary and showed 7 -h stability for ${ }^{99 m} \mathrm{Tc}-$ eluate, ${ }^{99 \mathrm{~m}} \mathrm{Tc}-\mathrm{HMDP}$, and ${ }^{99 \mathrm{~m}} \mathrm{Tc}-\mathrm{HSA}$ and 4 -h stability for 99m Tc-albumin nanocolloid according to those parameters.

\section{Visual Examination of IN-Stopper Connector}

No cores or plugs were observed on the vial caps, and the integrity of the obturator had not been affected after 30 punctures.

\section{Sterility Test}

The positive control and the positive transport sample showed microbiologic contamination in both media for all samples. For syringes withdrawn with the spinal needle and IN-Stopper, the negative control and the negative transport sample remained free of microbiologic contamination in both media. These results prove that syringes drawn with the spinal needle and IN-Stopper are microbiologically stable for up to $7 \mathrm{~h}$.

\section{Fingertip Radiation Exposure and Contamination}

The results showed an important reduction in the cumulative fingertip radiation dose for the dominant hand when the spinal needle and IN-Stopper was used, compared with the reversal method. Only the dominant hand received a dose during introduction of the system into the rubber cap. This dose remained low, compared with that received during the reversal procedure. There was no spillage of the radioactive solution during the tests, and no contamination was found. The results are summarized in Table 3 .

\section{DISCUSSION}

${ }^{99 m}$ Tc-radiopharmaceuticals are usually withdrawn by vial reversal. To avoid these reversal movements, long needles are ideal and allow for withdrawal of virtually all the preparation. We previously used Sterican needles $(60-\mathrm{mm}$, 23-gauge; B. Braun). Withdrawal was done by puncturing the vial cap several times to adjust the dose in order to obtain the prescribed activity. However, the needles were flexible and oscillated during removal of the vial's cap, creating radioactive microdroplets and exposure of the extremities of staff to radiation.

As a result, we finally chose a spinal needle to put into the vial's cap. To prevent air contamination of the preparation,

TABLE 3

Activity of ${ }^{99 m}$ Tc-Pertechnetate Eluate Vials and Dosimetry of Dominant and Nondominant Hands

\begin{tabular}{cccc}
\hline & & \multicolumn{2}{c}{ Mean dose \pm SD $(\mu S v / M B q)$} \\
\cline { 3 - 4 } Process & Range of vial activity $(\mathrm{MBq})$ & Dominant hand & Nondominant hand \\
\hline Routine disinfection of vial cap, reversal & $7,170-10,600$ & $12.3 \times 10^{-5} \pm 8.69 \times 10^{-5}$ & $17.5 \times 10^{-5} \pm 9.92 \times 10^{-5}$ \\
of vial, and drawing of syringe & & $6.65 \times 10^{-5} \pm 3.68 \times 10^{-5}$ & 0 \\
Insertion of spinal needle/IN-Stopper into vial & $10,218-11,800$ & $6.85 \times 10^{-5} \pm 6.81 \times 10^{-6}$ & $12.1 \times 10^{-5} \pm 4.89 \times 10^{-5}$ \\
Disinfection of obturator cap and syringe; & $6,790-11,215$ & & \\
withdrawal of spinal needle/IN-Stopper & & & \\
\hline
\end{tabular}


other teams tried to connect a bidirectional valve to the spinal needle, but this presents drawbacks such as the impossibility of use with a Luer slip syringe, an increase in exposure to radiation (7), and a risk that the work environment will be contaminated with radioactivity (8). IN-Stopper connectors were chosen because they allow use of both Luer slip and Luer-Lok slip syringes. Luer slip syringes are preferred by technologists for peripheral injections, but LuerLok slip syringes are preferred for securing central injections. The 23-gauge needle diameter allows for easy withdrawal and reduces the risk that rubber cores will form $(9,10)$. In our study, we proved that although the manufacturer recommended only 10 uses, the IN-Stopper connector could safely be used 30 times.

The conditions of the study followed routine practice. The ${ }^{99 m}$ Tc-radiopharmaceuticals tested were those most often prepared in the department. In fact, we dispense syringes for up

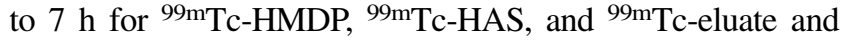
for up to $4 \mathrm{~h}$ for ${ }^{99 \mathrm{~m}} \mathrm{Tc}$-albumin nanocolloid. A maximum of 10 syringes could be withdrawn from one multidose preparation. Eluate withdrawal is necessary for ${ }^{99 \mathrm{~m} T c \text {-radiopharma- }}$ ceutical preparation. Thus, we also performed tests on the eluate. Levigoureux et al. had already proved the nonmicrobiologic contamination of multidose radiopharmaceuticals when the rules of hygiene are applied (11). Furthermore, the radioactive nature of radiopharmaceuticals cannot guarantee sterility (12). The study was performed under worst-case conditions: vials stored at room temperature and disinfection of the obturator cap only once for a syringe preparation. The cap obturator was not disinfected before dose adjustment and a new puncture.

Syringes were filled with air to allow aerobic microbial growth. Air was filtered to avoid external microbial contamination during the tests. Assessments on vials would have been easier, but to ensure the quality of the radiopharmaceuticals injected into the patient, microbiologic tests on syringes were preferred.

Concerning radiation safety, the system does not increase the fingertip dose even if there is an additional step: insertion of the spinal needle in the rubber cap before the first drawing. Furthermore, the nondominant hand does not touch the vial containing the radioactive solution.

The cost of the system (IN-Stopper obturator and spinal needle) is calculated at $€ 0.815$ (\$0.97), excluding taxes. This cost corresponds to an annual cost of less than $€ 1,300$ $(\$ 1,542.97)$ for 1,500 preparations.

Staff immediately accepted this new withdrawal method that improves ergonomics. Despite all these benefits, 2 risks remain: needle stick injury and internal radioactive contamination. These risks can be reduced by use of a needle recapper (13).
This study showed that eluate and preparation characteristics (visual and physicochemical) are not influenced by the system. Furthermore, we proved that administered syringes remained sterile and free from rubber coring.

\section{CONCLUSION}

When withdrawn with the spinal needle and IN-Stopper, ${ }^{99 \mathrm{~m}}$ Tc-eluate, ${ }^{99 \mathrm{~m}} \mathrm{Tc}-\mathrm{HMDP}$, and ${ }^{99 \mathrm{~m}} \mathrm{Tc}-\mathrm{HSA}$ syringes are guaranteed to be stable for up to $7 \mathrm{~h}$, and ${ }^{99 \mathrm{~m}} \mathrm{Tc}$-albumin nanocolloid is guaranteed for up to $4 \mathrm{~h}$. This method strikes a good balance among radiation safety, hygiene, and ergonomics. This method must be validated for other radiopharmaceuticals before widespread use.

\section{DISCLOSURE}

No potential conflict of interest relevant to this article was reported.

\section{REFERENCES}

1. IN-stopper. Europharmat website. http://base.euro-pharmat.com/pub_dm/fichedispositif-medical.aspx?id=14283. Updated August 27, 2012. Accessed December $21,2017$.

2. Hung JC, Ponto JA, Hammes RJ. Radiopharmaceutical-related pitfalls and artifacts. Semin Nucl Med. 1996;26:208-255.

3. Del Carmen Plancha-Mansanet M, Caballero-Calabuig E, Félix-Fontestad J,

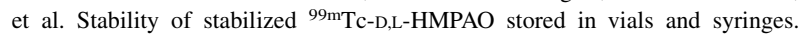
J Nucl Med Technol. 2008;36:213-216.

4. Zimmer AM, Pavel DG. Rapid miniaturized chromatographic qualitycontrol procedures for Tc-99m radiopharmaceuticals. J Nucl Med. 1977;18: $1230-1233$.

5. Robbins PJ. Chromatography of Technetium-99m Radiopharmaceuticals: A Practical Guide. Reston, VA: Society of Nuclear Medicine and Molecular Imaging; 1984.

6. General methods. 2.6 biological methods. 2.6 .1 sterility 07/2010:20601. In: European Pharmacopoeia. 8th ed. Strasbourg, France: Council of Europe; 2014

7. Galvez D, Cormier N, Blandin M, Renard PY, Froger S. Sécurisation de la préparation et de l'administration de médicaments radiopharmaceutiques (MRP): comparaison dosimétrique de valves bidirectionnelles. Europharmat congress website. http://www.euro-pharmat.com/communications-2016/download/938/1892/ 170. Presented 2016. Accessed December 21, 2017.

8. Le Garlantezec P, Rizzo-Padoin N, Aupee O, et al. Evaluation of the performance of transfer devices in a closed system using a radioactive solution of [ ${ }^{99 \mathrm{~m}} \mathrm{Tc}$ ] [in French]. Ann Pharm Fr. 2011;69:182-191.

9. Campagna R, Pessis E, Guerini H, et al. Occurrence of coring after needle insertion through a rubber stopper: study with prednisolone acetate. Eur Radiol. 2013;23:424-427.

10. Ponto JA. Self-sealing capacity of vial stoppers after multiple needle punctures. J Am Pharm Assoc (2003). 2013;53:58-60.

11. Levigoureux E, Hoffman A, Brun J, et al. Handling of radiopharmaceuticals drugs in hot cell: Implementation and validation of new hygiene procedures [in French]. Pharm Hosp Clin. 2012;47:106-115.

12. Jörg G, Fosselmann M, Leis W, et al. Inhibition of viability of microorganisms in $\left[{ }^{18} \mathrm{~F}\right]$-labeled radiopharmaceuticals. Nucl Med Biol. 2017;44:105-113.

13. Guidance on the recapping of needles in radiopharmacy and nuclear medicine. U.K. Radiopharmacy Group website. https://www.bnms.org.uk/images/ Recapping_needles_UKRG_guidance_v5_UKRG_format.pdf. Published 2013. Accessed December 212017. 